\title{
ANATOMÍA CAULINAR DE TRES ESPECIES DEL GÉNERO Hylocereus (Berger) Britton \& Rose (CACTACEAE) EN MÉXICO
}

\author{
STEM ANATOMY OF THREE SPECIES OF GENUS Hylocereus (Berger) Britton \& Rose \\ (CACTACEAE) IN MÉXICO
}

\author{
Ma. de los Ángeles García Aguilar ${ }^{1 *}$ y Teresa Terrazas² y Salvador Arias ${ }^{3}$
}

${ }^{1}$ Programa en Botánica, Campus Montecillo, Colegio de Postgraduados. Km 36.5 Carretera México-Texcoco. 56230, Montecillo, Texcoco, Edo. de México. Tel. y Fax. (55)5804 59 47, 59595202 47, (595) 9520200 Exts. 1300 y 1301. ²Departamento de Botánica y ${ }^{3}$ Jardín Botánico, Instituto de Biología, Universidad Nacional Autónoma de México. 04510 México, D. F., México.

\section{RESUMEN}

Se estudió la anatomía caulinar de tres especies del género Hylocereus (H. ocamponis, $H$. purpusii, $H$. undatus) con distribución en México, para evaluar la variación anatómica entre ellas y la relación de la madera con algunas variables de distribución geográfica (latitud, longitud, altitud) y climáticas (temperatura y precipitación). Se analizaron los sistemas dérmico, fundamental y vascular de 50 muestras de 10 poblaciones. Los tejidos primarios se evaluaron mediante la microtecnia convencional de inclusión en parafina, y para los tejidos secundarios se hicieron cortes con micrótomo de deslizamiento sin inclusión. A las variables cuantitativas se les aplicó un análisis de varianza y un análisis de comparación de medias (Tukey, 0.05). También se practicó un análisis de componentes principales donde se incluyeron tres variables geográficas y tres climáticas. Los resultados mostraron que las tres especies presentan elementos de vaso con placas de perforación simple y punteaduras alternas, fibras libriformes, parénquima paratraqueal escaso y radios heterogéneos; su madera es mesomórfica, característica de la subfamilia Cactoideae. Se detectaron diferencias $(P \leq \mathbf{0 . 0 5})$ entre especies en longitud del elemento de vaso, longitud de la fibra, altura y ancho de los radios. El análisis de componentes principales mostró que los caracteres de la madera asociados con la eficiencia en la conducción de agua, permitieron explicar el mayor porcentaje de la variación encontrada. Las tres especies presentaron mayor especialización en la eficiencia del transporte de agua que en el soporte mecánico.

Palabras clave: Hylocereus, anatomía caulinar, variación.

\section{SUMMARY}

The stem anatomy of three species of Hylocereus (H. ocamponis, $H$. purpusii, $H$. undatus) distributed in México was studied to evaluate the variation among species and the relationship of wood with distribution (latitude, longitude, elevation) and climate (temperature and rainfall) variables. Dermal, fundamental, and vascular tissues were analyzed on 50 samples from 10 populations. The primary tissues were embedded in paraffin while secondary tissues were sectioned with sliding microtome without embedding. Differences among populations or species were asserted by analysis of variance and by a mean comparison test (Tukey, 0.05). Wood variables plus three dis- tribution and three climate variables were included in a principal component analysis to identify those variables which explained the higher percentage of variance. The results showed that the three species of Hylocereus have vessel elements with simple perforation plates, pseudoscalariform intervascular pits, libriform fibers, scanty paratracheal parenchyma, and heterogenous rays; this wood is considered mesomorphic and distinctive of Cactoideae subfamily. There were differences $(P \leq \mathbf{0 . 0 5})$ among species for vessels element length, fiber length, and ray size. Principal component analysis showed that wood characters related to water movement had the highest contribution to explain variance, suggesting a trade-off between water movement efficiency and mechanical support.

Index words: Hylocereus, stem anatomy, variation.

\section{INTRODUCCIÓN}

El estudio anatómico de algunos caracteres de la madera y su variación se ha hecho principalmente en especies de importancia económica y de amplia distribución (Panshin y de Zeeuw, 1980) y ha sido esencial en clasificaciones de grupos taxonómicos importantes (Cronquist, 1981). A nivel específico la variación en estructuras de la madera se ha estudiado principalmente en función a gradientes de latitud y altitud, con respuesta diferencial (Noshiro et al., 1994; Aguilar-Rodríguez et al., 2006). Sin embargo, aunque en el estudio de la madera de la familia Cactaceae se han reconocido nueve líneas independientes de evolución (Mauseth y Plemons-Rodríguez, 1998), aún es escaso el conocimiento de otros rasgos anatómicos caulinares en muchos géneros (Terrazas y Arias, 2002); y más escasos aún los relacionados a los patrones de variación de la madera en especies de cactáceas de amplia distribución (Arias y Terrazas, 2001). 
En el género Hylocereus las descripciones anatómicas han centrado su atención en $H$. undatus, especie que tiene amplia variación morfológica, fisiológica y genética (Nerd y Mizrahi, 1997). Actualmente no se cuenta con descripciones anatómicas que permitan apoyar el reconocimiento de las especies del género Hylocereus presentes en México y su respectiva distribución. Por ello el objetivo de esta investigación es contribuir al conocimiento de la anatomía caulinar de Hylocereus ocamponis, $H$. purpusii y $H$. undatus presentes en México, así como evaluar si algunos caracteres de la madera asociados con el transporte del agua y el soporte mecánico se relacionan con su distribución geográfica (latitud, longitud, altitud) y con las condiciones climáticas (temperatura máxima, temperatura mínima y precipitación promedio anual).

\section{MATERIALES Y MÉTODOS}

La recolecta del material se hizo en 10 poblaciones silvestres de Hylocereus ocamponis (cuatro poblaciones), $H$. purpusii (cuatro) y H. undatus (dos) en los Estados de Nayarit, Michoacán, Guerrero y Chiapas (Cuadro 1). En cada localidad se muestrearon de tres a cinco individuos fenológicamente maduros de cada especie. Los ejemplares de respaldo se depositaron en el herbario CHAPA del Colegio de Postgraduados, Campus Montecillo. Se tomaron segmentos $(2 \mathrm{~cm}$ de longitud) de ramas adultas de la parte apical, media y basal de cada individuo y se fijaron en una solución de glicerina-etanol-agua (1:1:1). Para la descripción del tejido primario se utilizó la parte media de la rama; para el tejido vascular secundario y la peridermis se utilizó la parte basal de cada individuo; la parte apical permitió entender el desarrollo de la epidermis y del tejido vascular. Para la obtención de preparaciones permanentes, las muestras de la parte apical y media se procesaron con la microtecnia convencional de inclusión en parafina. Los tejidos se cortaron a un grosor de $14 \mu \mathrm{m}$ con un micrótomo rotatorio (Leica RM2125®), se tiñeron y montaron en resina sintética de acuerdo con Johansen (1940).

Cuadro 1. Datos de las localidades de procedencia de las tres especies del género Hylocereus en México. Los ejemplares de herbario se depositaron en el herbario CHAPA del Colegio de Postgraduados, Campus Montecillo.

\begin{tabular}{llcc}
\hline Especie & Localidad y número de colecta & $\begin{array}{c}\text { Número de } \\
\text { individuos } \\
\text { colectados }\end{array}$ & $\begin{array}{c}\text { Latitud N } \\
\text { (m) }\end{array}$
\end{tabular}

\begin{tabular}{|c|c|c|c|c|c|}
\hline H. purpusii & Nayarit, Ixtlán del Río, García-Aguilar 21 & 5 & $21^{\circ} 02^{\prime} 30^{\prime \prime}$ & $104^{\circ} 13^{\prime} 45^{\prime \prime}$ & 15 \\
\hline H. purpusii & Nayarit, Syngaita, García-Aguilar 26 & 5 & $21^{\circ} 34^{\prime} 18^{\prime \prime}$ & $105^{\circ} 14^{\prime} 17^{\prime \prime}$ & 31 \\
\hline H. purpusii & Nayarit, Jala, García-Aguilar 22 & 5 & $21^{\circ} 32^{\prime} 30^{\prime \prime}$ & $104^{\circ} 27^{\prime} 09^{\prime \prime}$ & 1132 \\
\hline H. undatus & Michoacán, La Piedad, García-Aguilar 20 & 4 & $20^{\circ} 30^{\prime \prime} 32^{\prime \prime}$ & $102^{\circ} 52^{\prime} 14^{\prime \prime}$ & 2262 \\
\hline H. ocamponis & Michoacán, Cerro Colorado, García-Aguilar 35 & 3 & $19^{\circ} 18^{\prime} 51^{\prime \prime}$ & $100^{\circ} 28^{\prime} 12^{\prime \prime}$ & 1195 \\
\hline H. ocamponis & Michoacán, El Zapote, García-Aguilar 34 & 4 & $19^{\circ} 55^{\prime} 27^{\prime \prime}$ & $101^{\circ} 03^{\prime} 15^{\prime}$, & 796 \\
\hline H. ocamponis & Michoacán, Rosas Moradas, García-Aguilar 36 & 3 & $18^{\circ} 39^{\prime} 38^{\prime \prime}$ & $103^{\circ} 26^{\prime} 53^{\prime \prime}$ & 1029 \\
\hline H. ocamponis & Michoacán, Arteaga, García-Aguilar 37 & 3 & $18^{\circ} 23^{\prime} 03^{\prime \prime}$ & $102^{\circ} 22^{\prime} 34^{\prime \prime}$ & 710 \\
\hline H. purpusii & Guerrero, Salitrera, García-Aguilar 32 & 5 & $17^{\circ} 53^{\prime} 43^{\prime \prime}$ & $101^{\circ} 21^{\prime} 11^{\prime \prime}$ & 433 \\
\hline H. undatus & Chiapas, Ocozocoautla, García-Aguilar 17 & 3 & $16^{\circ} 76^{\prime}$ & $93^{\circ}$ & 820 \\
\hline
\end{tabular}


Para la descripción del xilema secundario, la parte basal del tallo se cortó con un micrótomo de deslizamiento (Leica SM2400®) en secciones transversales y tangenciales a un grosor de $18 \mu \mathrm{m}$, se tiñeron con safranina y se montaron en resina sintética. Para la obtención de disociados de madera, se obtuvieron porciones de la madera cercanas al cámbium vascular de $0.5 \times 0.5 \mathrm{~cm}$, las cuales se colocaron en una solución de Jeffrey (Johansen, 1940) y se sometieron a una temperatura de $50{ }^{\circ} \mathrm{C}$ en una estufa de secado por $24 \mathrm{~h}$. El material disociado se lavó y se hicieron preparaciones temporales para cuantificar la longitud de los elementos de vaso y fibras. Para la descripción del xilema secundario se siguieron las recomendaciones de la Asociación Internacional de los Anatomistas de la Madera (IAWA, 1989); los radios se clasificaron conforme a los criterios de Kribs (1935).

Se hicieron 50 mediciones por individuo con un analizador de imágenes (IMAGE-Pro Plus® versión 3.1; Media Cybernetics, 1997) adaptado a un microscopio Olympus BX-50®. Se calculó la conductividad relativa para cada especie (Zimmermann, 1983) mediante la ecuación de Poiseuille modificada por Fahn et al. (1986): $\mathrm{CR}=\left(\mathrm{R}^{4}\right)$ (FRE), donde $\mathrm{R}$ es el radio del elemento de vaso, y FRE el número de vasos $/ \mathrm{mm}^{2}$. También se calculó el índice de vulnerabilidad (Carlquist, 1977): IV = DV/FRE, donde DV es el diámetro del elemento de vaso, y FRE el número de vasos $/ \mathrm{mm}^{2}$, y el índice de Chattaway (1936): IC = LFIB/LVAS, donde LFIB es la longitud de la fibra y LVAS es la longitud del elemento de vaso.

A cada variable se le aplicó un análisis de varianza, seguido por un análisis de comparación de medias (Tukey, $0.05)$ para detectar diferencias significativas entre especies para los caracteres cuantificados. Se hizo un análisis de correlación entre las variables anatómicas y algunas variables geográficas (latitud, longitud y altitud) y climáticas (temperatura máxima, temperatura mínima y precipitación promedio anual). También se practicó un análisis de componentes principales (ACP) con tres variables geográficas, tres climáticas y 18 variables anatómicas, para explicar la variación en las especies (SAS Institute, 1989).

\section{RESULTADOS}

\section{Tejido primario}

La cutícula es lisa en las tres especies estudiadas (Figura 1A), con un espesor que varía de $8.01 \pm 1.44 \mu \mathrm{m}$ en $H$. purpusii hasta $10.94 \pm 1.26 \mu \mathrm{m}$ en $H$. ocamponis (Cuadro 2). La epidermis es simple, cuyas células presentan forma rectangular en corte transversal, con cristales de oxalato de calcio de forma prismática, comúnmente de uno a tres cristales por célula en todas las especies. La hipo- dermis es colenquimatosa (Figura 1B, C), con estratos que varían de dos a cuatro, con un grosor de $130.91 \pm 30.27$ $\mu \mathrm{m}$ en $H$. undatus hasta $196.85 \pm 14.90 \mu \mathrm{m}$ en $H$. ocamponis.

Cuadro 2. Grosor de cutícula e hipodermis de tres especies del género Hylocereus en México.

\begin{tabular}{lcc}
\hline Especie & Cutícula $(\mu \mathrm{m})$ & Hipodermis $(\mu \mathrm{m})$ \\
\hline H. ocamponis & $10.94 \pm 1.26$ & $196.85 \pm 14.90$ \\
H. purpusii & $8.01 \pm 1.44$ & $149.04 \pm 37.66$ \\
H. undatus & $9.28 \pm 1.17$ & $130.91 \pm 30.27$
\end{tabular}

Los datos son promedios \pm el error estándar de la media $(\mathrm{n}=50)$.

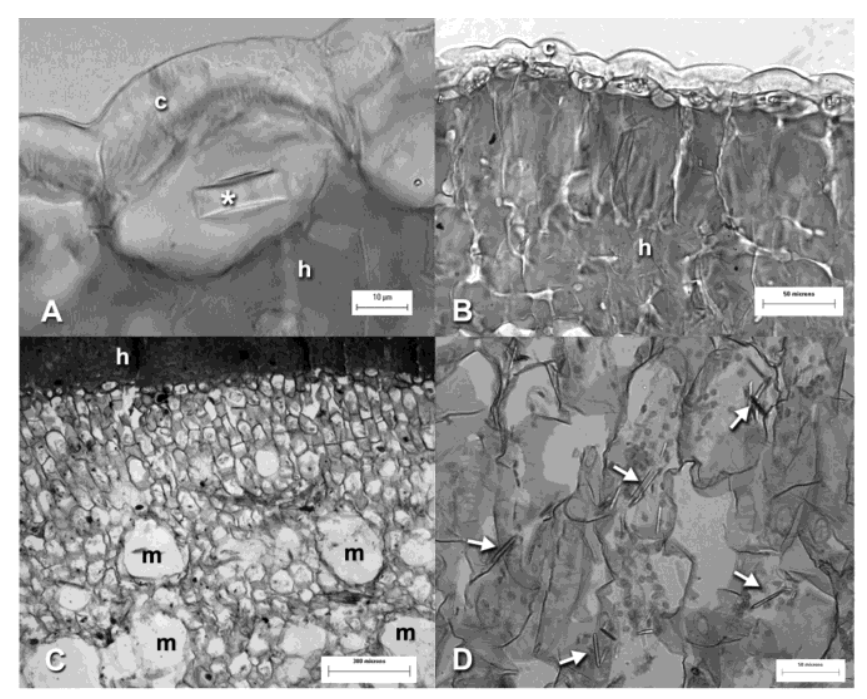

Figura 1. Cutícula, hipodermis y corteza primaria de tres especies del género Hylocereus. Cortes transversales. A. Cutícula lisa, H. ocamponis. B. Hipodermis colenquimatosa, $H$. undatus. C. Parénquima reservante con células mucílago, $H$. purpusii. D. Región de parénquima reservante con cristales lenticulares, $H$. purpusii. Abreviaturas: $\mathbf{c}=$ cutícula, $\mathbf{h}=$ hipodermis, $\mathbf{m}=$ mucílago. $*=$ cristal prismático, flechas $=$ cristales lenticulares. Barras: $A=10 \mu \mathrm{m}, \mathrm{B}, \mathrm{D}=$ $50 \mu \mathrm{m}, \mathrm{C}=100 \mu \mathrm{m}$.

En la corteza primaria se observaron dos regiones, una de clorénquima y otra de parénquima reservante; la primera con células de parénquima en empalizada de tres a cinco hileras, que al alejarse de la hipodermis pierden su arreglo. La región reservante está conformada de células de parénquima e idioblastos redondos con mucílago, en todas las especies (Figura 1C), así como cristales lenticulares (Figura 1D), aciculares y areniscas que se depositan en el lumen de algunas células del parénquima. Los haces vasculares corticales son de tamaño variable y con crecimiento secundario, que se observaron principalmente en la región de parénquima reservante (Figura 2A). En las tres especies 
se encuentran abundantes fibras adyacentes al floema primario desde la región apical de las ramas (Figura 2B). La médula presenta células de parénquima de forma isodiamétrica con granos de almidón, sin presencia de haces vasculares medulares en estas especies estudiadas.

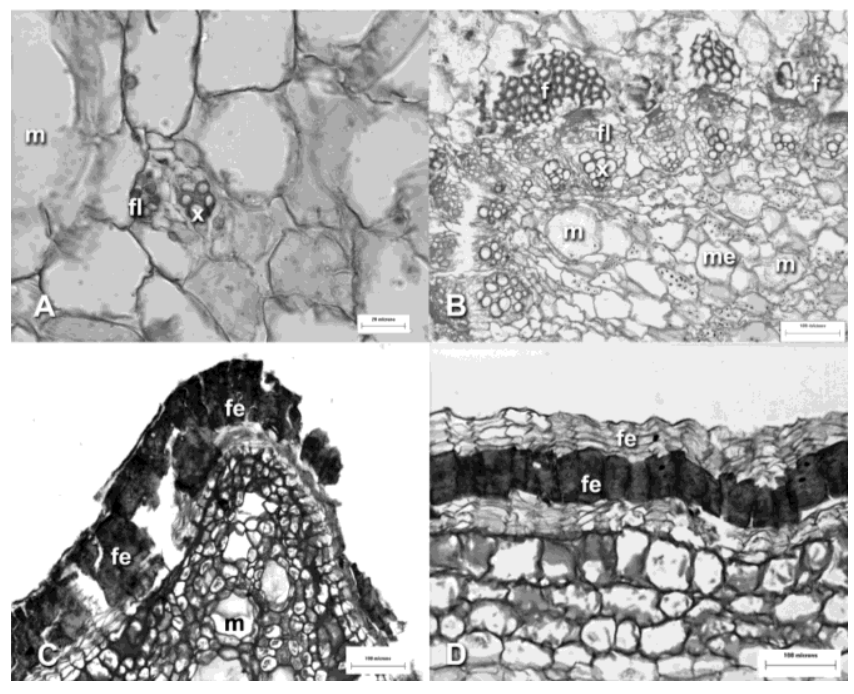

Figura 2. Corteza primaria y peridermis de tres especies del género Hylocereus. A. Haz vascular cortical en la región de parénquima reservante, $\boldsymbol{H}$. ocamponis. B. Fibras sobre floema, $\boldsymbol{H}$. undatus. C. Peridermis arista de costilla, $\boldsymbol{H}$. purpusii. D. Peridermis base tallo, $\boldsymbol{H}$. purpusii. Abreviaturas: $\mathrm{f}=$ fibra, $\mathrm{fe}=$ felema, $\mathrm{fl}=$ floema, $\mathrm{m}=$ mucílago, $\mathrm{me}=$ médula, $x=$ xilema, Barras: $A=20 \mu \mathrm{m} . \mathrm{B}-\mathrm{D}=100$ $\mu \mathrm{m}$.

\section{Tejido secundario}

Peridermis. En la región apical del tallo las células epidérmicas sufren divisiones periclinales desde el inicio del desarrollo, lo que origina una peridermis unidireccional, por lo que se forman de uno más de seis estratos de células de felema en las aristas de las costillas desde la región cercana al meristemo apical (Figura 2C). Además, la epidermis es también reemplazada por una peridermis unidireccional en la parte basal del tallo. El felógeno se origina de las células epidérmicas en las elevaciones de las costillas y se extiende hacia las depresiones de las mismas. El felema se caracteriza por tener células de paredes delgadas que se alternan con células de paredes gruesas (Figura 2D).

Xilema secundario. Los anillos de crecimiento son inconspicuos para las tres especies. La porosidad es difusa (Figura 3A) con una densidad de 23 vasos $/ \mathrm{mm}^{2}$ en $H$. ocamponis hasta 44 vasos $/ \mathrm{mm}^{2}$ en $H$. undatus. Más de 70 $\%$ de los vasos se arreglan en grupos de dos a cuatro, y el resto son solitarios. Los vasos son comúnmente circulares (Figura 3A), con un diámetro tangencial medio de 31.86 $\pm 0.99 \mu \mathrm{m}$ en $H$. purpusii hasta $38.91 \pm 1.51 \mu \mathrm{m}$ en $H$. undatus. La longitud media de los elementos de vaso es de $211.13 \mu \pm 5.77 \mu \mathrm{m}$ en $H$. undatus hasta $246.35 \pm 3.21$ $\mu \mathrm{m}$ en $H$. purpusii. Presenta placas de perforación simples $\mathrm{y}$ punteaduras intervasculares alternas escalariformes $\mathrm{y}$ pseudoescalariformes (Figura 3D).

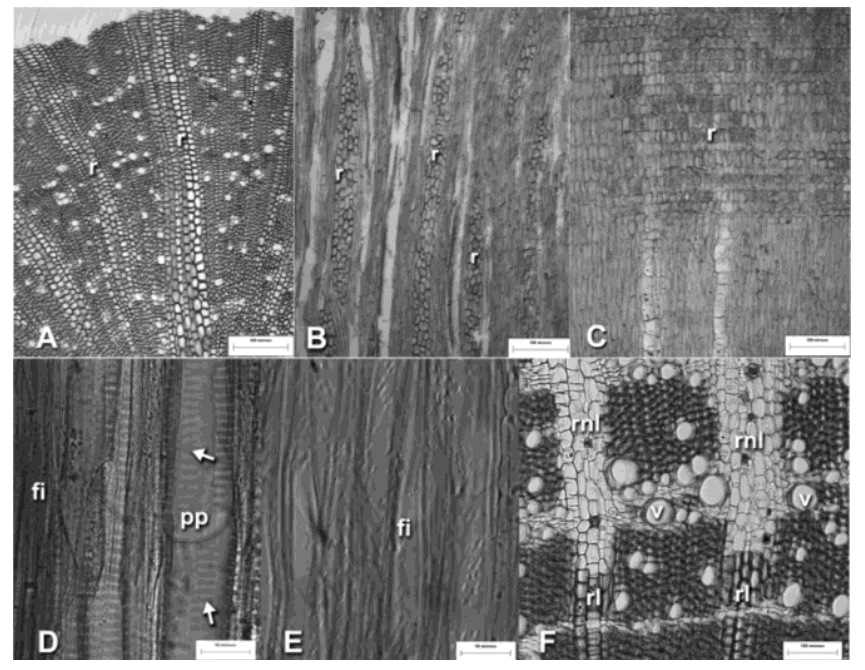

Figura 3. Xilema secundario de tres especies del género Hylocereus. A. Corte transversal, porosidad difusa con abundantes vasos solitarios, $\boldsymbol{H}$. undatus. B. Corte tangencial, radios multiseriados, $H$. undatus. C. Corte radial, células erectas y procumbentes del radio, $\boldsymbol{H}$. ocamponis. D. Corte tangencial, elemento de vaso con placa simple y punteaduras alternas pseudoescalariformes, $\boldsymbol{H}$ purpusii. E. Corte radial, fibras libriformes, $\boldsymbol{H}$. ocamponis. $\mathbf{F}$. Corte transversal, radios no lignificados y lignificados, $H$. purpusii. Abreviaturas: $f i=$ fibra, $p p$ $=$ placa de perforación, $\mathbf{r}=$ radio, $\mathbf{r l}=$ radio lignificado, $\mathbf{r n l}=\mathbf{r a}-$ dio no lignificado, $\mathrm{v}=$ vaso. Barras: $\mathrm{A}-\mathrm{C}=\mathbf{3 0 0} \mu \mathrm{m}, \mathrm{D}, \mathrm{E}=\mathbf{5 0} \mu \mathrm{m} \mathrm{F}$ $=100 \mu \mathrm{m}$.

El índice de vulnerabilidad adquiere valores menores a 0.83 en $H$. purpusii y superiores a 1.73 en $H$. undatus. Las fibras son libriformes con punteaduras simples en la cara radial (Figura 3E), con una longitud de $423.48 \pm$ $10.17 \mu \mathrm{m}$ en $H$. undatus y de $541.14 \pm 3.21 \mu \mathrm{m}$ en $H$. purpusii. El diámetro de la fibras es de $13.40 \pm 0.25 \mu \mathrm{m}$ en $H$. ocamponis y de $14.15 \pm 0.22 \mu \mathrm{m}$ en $H$. purpusii. El parénquima axial es paratraqueal escaso (Figura $3 \mathrm{~F}$ ), con tres células por serie parenquimatosa sin contenidos celulares, aunque en las regiones no lignificadas el parénquima axial puede estar en bandas. Los radios son heterogéneos para las especies estudiadas (Figura 3B), con alturas de $1140 \pm 0.131 \mu \mathrm{m}$ en $H$. undatus hasta $2730 \pm 1.03 \mu \mathrm{m}$ en $H$. purpusii, y de dos a nueve células de ancho. Las células de radio son erectas cortas y procumbentes (Figura 3C); algunas células de los radios se lignifican tardíamente (Figura 3F). 


\section{Variación poblacional}

Se detectaron diferencias entre las poblaciones muestreadas para los atributos de espesor de la cutícula ( $\mathrm{P} \leq$ $0.0001)$, hipodermis $(\mathrm{P} \leq 0.0001)$, longitud del elemento de vaso $(\mathrm{P} \leq 0.0001)$, longitud de la fibra $(\mathrm{P} \leq 0.0001)$ y la altura y ancho de los radios $(\mathrm{P} \leq 0.0001)$.

El análisis de correlación entre latitud, longitud, temperatura y precipitación y los 14 caracteres anatómicos analizados reveló que la densidad de vasos correlaciona positivamente con latitud, la altura de radio con longitud, y negativamente el ancho de los radios con longitud, la densidad de vasos y la longitud de la fibra con altitud (Cuadro 3). La correlación entre la altura de radios y la temperatura máxima anual fue de $\mathrm{r}_{\mathrm{s}}=0.43(\mathrm{P} \leq 0.0001)$, y entre el ancho de los radios y la precipitación promedio anual fue $\mathrm{r}_{\mathrm{s}}=-0.32(\mathrm{P} \leq 0.0001)$ (Cuadro 4).
El análisis de componentes principales mostró que cuatro componentes explican $72.5 \%$ de la variación total (Cuadro 5), donde el primer componente con un valor característico de 4.61 explica $28.6 \%$ de la varianza total. Las variables de mayor peso fueron el diámetro del elemento de vaso y el índice de vulnerabilidad. El segundo componente explicó $18.5 \%$ de la varianza residual, en cuyo caso la densidad de los elementos de vaso, el CR e IV contribuyeron a explicar dicha variación. El tercer componente explicó $13.6 \%$ de la variación remanente, donde las variables de mayor peso fueron diámetro del lumen, grosor de la pared de la fibra y la altura de los radios. El cuarto componente explicó $11.5 \%$ de la variación residual, donde la longitud de los elementos de vaso, longitud de la fibra e IC contribuyeron a explicar dicha variación (Cuadro $5)$.

Cuadro 3. Coeficientes de correlación entre latitud, longitud y altitud y 14 caracteres anatómicos de tres especies del género Hylocereus en México.

\begin{tabular}{|c|c|c|c|}
\hline Carácter anatómico & Latitud & Longitud & Altitud \\
\hline Cutícula & $0.38^{* *}$ & 0.05 & -0.15 \\
\hline Hipodermis & 0.23 & 0.08 & 0.07 \\
\hline Longitud del elemento de vaso & 0.08 & 0.24 & -0.06 \\
\hline Densidad de vasos & $0.31 * *$ & 0.19 & $-0.25^{* *}$ \\
\hline Longitud de fibra & 0.03 & 0.27 & $-0.32 * *$ \\
\hline Diámetro de fibra & 0.04 & 0.02 & 0.01 \\
\hline Grosor de la pared de fibra & 0.11 & 0.06 & -0.07 \\
\hline Altura de radio & 0.18 & $0.39 * *$ & -0.19 \\
\hline Ancho de radio & -0.13 & $-0.30 * *$ & 0.07 \\
\hline Conductividad relativa (CR) & 0.13 & 0.05 & -0.01 \\
\hline Índice de vulnerabilidad (IV) & -0.26 & -0.17 & 0.15 \\
\hline Índice de Chattaway (IC) & 0.20 & 0.02 & -0.16 \\
\hline
\end{tabular}

$* *=\mathrm{P} \leq 0.0001$

Cuadro 4. Coeficientes de correlación entre 14 caracteres anatómicos de tres especies del género Hylocereus en México y variables de temperatura y precipitación.

\begin{tabular}{|c|c|c|c|}
\hline Carácter anatómico & $\begin{array}{c}\text { Temperatura máxima } \\
\left({ }^{\circ} \mathrm{C}\right)\end{array}$ & $\begin{array}{l}\text { Temperatura } \\
\text { mínima }\left({ }^{\circ} \mathrm{C}\right)\end{array}$ & Precipitación promedio anual (mm) \\
\hline Hipodermis & 0.18 & -0.18 & 0.12 \\
\hline Diámetro del elemento de vaso & 0.01 & -0.03 & -0.04 \\
\hline Densidad de vasos & 0.10 & -0.07 & 0.00 \\
\hline Longitud de fibra & 0.12 & 0.04 & 0.14 \\
\hline Grosor de la pared de fibra & -0.01 & -0.05 & 0.09 \\
\hline Altura de radio & $0.43 * *$ & 0.16 & 0.25 \\
\hline Ancho de radio & -0.15 & 0.02 & $-0.32 * *$ \\
\hline Conductividad relativa (CR) & 0.06 & -0.08 & -0.00 \\
\hline Índice de vulnerabilidad (IV) & -0.03 & 0.02 & -0.06 \\
\hline
\end{tabular}

$* *=\mathrm{P}<0.0001$ 
Cuadro 5. Vectores característicos correspondientes al análisis de componentes entre doce caracteres anatómicos de tres especies del género Hylocereus en México.

\begin{tabular}{|c|c|c|c|c|}
\hline Caracteres anatómicos & $\begin{array}{c}\text { Componente } \\
1 \\
\end{array}$ & $\begin{array}{c}\text { Componente } \\
2 \\
\end{array}$ & $\begin{array}{c}\text { Componente } \\
3 \\
\end{array}$ & $\begin{array}{c}\text { Componente } \\
4 \\
\end{array}$ \\
\hline Valor característico & 4.61 & 2.97 & 2.17 & 1.84 \\
\hline Longitud del elemento de vaso & -0.001 & 0.081 & 0.201 & -0.503 \\
\hline Densidad de vasos & -0.122 & 0.535 & 0.138 & -0.023 \\
\hline Longitud de fibra & -0.153 & 0.111 & 0.111 & 0.430 \\
\hline Diámetro de fibra & 0.112 & -0.170 & 0.601 & 0.109 \\
\hline Altura de radio & -0.094 & 0.064 & 0.219 & -0.146 \\
\hline Ancho de radio & -0.032 & 0.131 & -0.122 & 0.100 \\
\hline Conductividad relativa (CR) & 0.280 & 0.450 & 0.060 & 0.044 \\
\hline Índice de vulnerabilidad (IV) & 0.396 & -0.251 & -0.120 & 0.031 \\
\hline Índice de Chattaway (IC) & -0.119 & 0.028 & -0.056 & 0.697 \\
\hline
\end{tabular}

${ }^{\dagger}$ En negritas las variables con mayor carga en cada componente

\section{DISCUSIÓN}

La epidermis es simple, con presencia de uno a tres cristales prismáticos por célula, igual que para especies de Cephalocereus y Neobuxbamia de la subfamilia Cactoideae (Terrazas y Mauseth, 2002). La cutícula es lisa, cuyo valor más alto se encontró en $H$. ocamponis, y hubo una relación significativa entre el grosor de cutícula y la latitud. Las especies estudiadas presentaron una hipodermis colenquimatosa y multiestratificada, característica en miembros de la subfamilia Cactoideae (Gibson y Horak, 1978; LozaCornejo y Terrazas, 2003).

En la corteza primaria se observaron dos regiones, una de clorénquima y otra de parénquima reservante con idioblastos con mucílago y haces vasculares corticales con fibras sobre floema primario en la parte apical de la rama, caracteres típicos de la subfamilia Cactoideae (Sajeva y Mauseth, 1991). En la médula se observaron células de parénquima y granos de almidón; Terrazas y Mauseth (2002) mencionan que la presencia de haces vasculares medulares es característica de esta subfamilia, pero en estas especies estudiadas no están presentes.

La peridermis de las especies del género Hylocereus correspondió a lo reportado en otras especies de Cactoideae, de origen epidérmico y con desarrollo unidireccional (Terrazas y Arias, 2002). En las especies estudiadas la peridermis se establece desde el inicio del desarrollo donde comienza en las aristas de las costillas, y se extiende gradualmente hacia los valles de las mismas, aunque en algunos ejemplares llega a ser visible hasta muy avanzado el desarrollo.
La madera de las tres especies estudiadas del género Hylocereus con distribución en México, presentó elementos de vaso con placas de perforación simple y punteaduras alternas pseudoescalariformes, parénquima paratraqueal escaso y radios heterogéneos, mismos que son característicos de la subfamilia Cactoideae (Gibson, 1973; Mauseth y Plemons-Rodríguez, 1998; Terrazas y Mauseth, 2002). Su madera es mesomórfica; es decir, sin el patrón característico de las especies que se desarrollan en sitios con limitantes de agua (Carlquist, 1988). La longitud del elemento de vaso varió entre 211 y $242 \mu \mathrm{m}$, valores más altos a los señalados por Gibson (1973) como promedios para la subfamilia Cactoideae entre 114 y $137 \mu \mathrm{m}$; aunque clasificados como cortos por Van der Graaff y Baas (1974) y Chalk (1989).

Se encontraron fibras libriformes, características también de la subfamilia Cactoideae (Terrazas y Mauseth, 2002). La longitud de las fibras varió de 423 a $541 \mu \mathrm{m}$, consideradas como cortas en comparación con otras dicotiledóneas (Chalk, 1989). Se observó una correlación significativa entre longitud, lumen y grosor del mismo elemento, asociación que coincide con lo encontrado en otras especies de cactáceas (Bernal-Salazar y Terrazas, 2005). Para la subfamilia Cactoideae se ha registrado una relación alométrica entre el tamaño de los elementos traqueales (elementos de vaso y fibras) y la altura de los individuos, puesto que las especies más altas presentan elementos traqueales más largos y anchos, mientras que la especies de talla corta tienden a presentar elementos cortos y angostos (Gibson, 1973; Gibson y Nobel, 1986; Terrazas y LozaCornejo, 2003). Sin embargo, esta relación no fue observada en las especies del género Hylocereus; en cambio se 
encontró una correlación entre la longitud de los elementos traqueales y algunas variables geográficas, correlación que fue moderada con la longitud y una de las más altas con la altitud.

El índice de Chatttaway mostró valores menores de 2.6 en todas las localidades muestreadas; según Carlquist (1988), las especies que presentan valores mayores o iguales a 2.6 son las que se especializan en alcanzar niveles óptimos del sistema mecánico y por tanto forman fibras largas. Este comportamiento también se explica por el hábito de crecimiento de las especies de Hylocereus aquí estudiadas. El parénquima axial es paratraqueal escaso, característico en las maderas fibrosas de las especies de Cactoideae (Mauseth y Plemons-Rodríguez, 1988; Terrazas y Mauseth, 2002). Las tres especies presentaron radios altos y anchos según la clasificación de Kribs (1935), con $H$. ocamponis como la especie con los radios más altos y angostos, en tanto que $H$. undatus presentó los radios más cortos y anchos. Hubo una correlación de $\mathrm{r}_{\mathrm{s}}=0.39$ ( $\mathrm{P} \leq$ 0.0001 ) entre altura de los radios y su longitud; así como entre el ancho de los radios y la temperatura; estas correlaciones difieren de lo registrado para Pachycereus pectenaboriginum, especie en la que el tamaño de los radios se asocia significativamente con la altitud (Arias y Terrazas, 2001).

El análisis de componentes principales mostró que los caracteres de la madera asociados con la eficiencia en la conducción de agua (lumen del elemento del vaso, densidad de vasos); así como soporte mecánico (diámetro, lumen y grosor de la pared de la fibra), mismos que determinan los índices de CR e IV, fueron los caracteres que permitieron explicar el mayor porcentaje de la variación encontrada en las tres especies estudiadas.

En las diferencias significativas registradas entre localidades, los sitios Syngaita, Ixtlán del Río y Salitrera presentaron los valores más altos en longitud del elemento de vaso, longitud de la fibra, e IV, cuyos individuos correspondieron a $H$. purpusii. Los individuos recolectados en la localidad Rosas Moradas presentaron los valores más altos en diámetro del elemento de vaso y correspondieron a $H$. ocamponis. Los individuos recolectados en la localidad de La Piedad registraron los valores más bajos de longitud de la fibra y corresponden a $H$. untadus. Otro carácter anatómico que ayudó en menor grado a explicar la variación de la madera fue el ancho de los radios, ya que la especie $H$. undatus presentó los radios más anchos y cortos de las tres especies aquí estudiadas.

El análisis de componentes principales reveló que el índice de conductividad relativa e índice de vulnerabilidad son los atributos anatómicos que más contribuyen a expli- car la variación en estas tres especies, lo que sugiere que las especies del género Hylocereus presentan especialización en la eficiencia del transporte de agua, en comparación con el soporte mecánico, debido a sus hábitos de crecimiento epífitos o hemiepífitos.

\section{CONCLUSIONES}

Existen atributos en la anatomía caulinar, como grosor de cutícula, longitud y diámetro del elemento de vaso, longitud de fibra, así como ancho y alto de radios, y los índices de vulnerabilidad y de Chattaway, que permiten explicar la variación de las tres especies del género Hylocereus presentes en México.

Tres atributos anatómicos caulinares (grosor de cutícula, densidad de vasos y tamaño de radios) se relacionaron con algunas variables geográficas, por lo que podrían contribuir al conocimiento de los patrones de su distribución en México.

\section{AGRADECIMIENTOS}

Al Consejo Nacional de Ciencia y Tecnología por el apoyo a la beca para realizar estudios de posgrado (185128). Al Colegio de Postgraduados por las facilidades en el trabajo de anatomía y a Julio César Monteros del Instituto de Biología, UNAM, por el diseño de imágenes.

\section{BIBLIOGRAFÍA}

Aguilar-Rodríguez S, T Terrazas, L López-Mata (2006) Anatomical wood variation of Buddleja cordata (Buddlejaceae) along its natural range in Mexico. Trees 20:253-261.

Arias S, T Terrazas (2001) Variación en la anatomía de la madera de Pachycereus pecten-aboriginum (Cactaceae). Anales Inst. Biol. Univ. Nac. Auton. México, Bot. 72:157-169.

Bernal-Salazar S, T Terrazas (2005) Wood anatomical variation of Neobuxbaumia tetetzo: A columnar Cactaceae. J. Arid Environ. 63:671-685.

Carlquist S (1977) Ecological factors in wood evolution: a floristic approach. Amer. J. Bot. 64:887-896

Carlquist S (1988) Comparative Wood Anatomy. Systematic, Ecological, and Evolutionary Aspects of Dicotyledon Wood . SpringerVerlang. Berlín. Alemania. $436 \mathrm{p}$.

Chalk L (1989) The effects of ecological conditions on wood anatomy. In: Anatomy of Dicotyledons. Vol. II. C R Metcalfe, L Chalk (eds). Clarendon, Oxford, UK. pp:152-156.

Chattaway M M (1936) The relation between fibre and cambial initial length in dicotyledonous woods. Trop. Woods 46:16-20.

Cronquist A (1981) An Integrated System of Classification of Flowering Plants. Columbia University Press, New York. 1262 p.

Fahn A, E Werker, P Bass (1986) Wood Anatomy and Identification of Trees and Shrubs from Israel and Adjacent Regions. Israel Academy of Sciences, Jerusalem. $221 \mathrm{p}$.

Gibson A C (1973) Comparative anatomy of secondary xylem in Cactoideae (Cactaceae). Biotropica 5:29-65. 
Gibson AC, K E Horak (1978) Systematic anatomy and phylogeny of Mexican columnar cacti. Ann. Missouri Bot. Gard. 65:9991057.

Gibson A C, P S Nobel (1986) The Cactus Primer. Harvard University Press. Cambridge Massachussets, USA. $286 \mathrm{p}$.

IAWA Committe (1989) IAWA list of microscopic features for hardwood identification IAWA Bull. n.s. 10:219-332.

Johansen J (1940) Plant Microtechnique. McGraw-Hill Book Company. New York. USA. 491 p.

Kribs D A (1935) Salient lines of specialization in the wood rays of dicotyledons. Bot. Gaz. 96:547-557.

Loza-Cornejo S, T Terrazas (2003) Epidermal and hypodermal characteristics in North American Cactoideae (Cactaceae). J. Plant Res. 116:27-35

Mauseth J D, J Plemons-Rodríguez (1998) Evolution of extreme xeromorphic characters in wood: A study of nine evolutionary lines in Cactaceae. Amer. J. Bot. 84:209-218.

Media Cybernetics (1997) Image-Pro Plus Reference Guide for Windows. Silver Spring, Maryland. $487 \mathrm{p}$.

Nerd A, Y Mizrahi (1997) Reproductive biology of cactus fruit crops. Hort. Rev. 18:321-346.
Noshiro S L, L Joshi, M Suzuki (1994) Ecological wood anatomy of Alnus nepalensis (Betulaceae) in East Nepal. J. Plant Res. 107:399-408.

Panshin A J, C de Zeeuw (1980) Textbook of Wood Technology. 4th ed. McGraw-Hill. New. York, USA. 722 p.

Sajeva M, D Mauseth (1991) Leaf-like structure in the photosynthetic, succulent stems of cacti. Ann. Bot. 68:405-411.

SAS Institute (1989) SAS Procedure Guide. Release 6.03. SAS Institute, Cary, North Carolina, USA.

Terrazas T, S Arias (2002) Comparative stem anatomy in the subfamily Cactoideae. Bot. Rev. 68:444-473.

Terrazas T, J D Mauseth (2002) Shoot anatomy and morphology. In: Cacti: Biology and Uses. P S Nobel (ed). University of California Press. Berkeley, California, USA. pp:23-40.

Terrazas T, S Loza-Cornejo (2003) Anatomía de la madera y alometría de veinte especies de Stenocereus (Cactaceae). Anales Inst. Biol. Univ. Nac. Auton. México, Bot. 74:195-208.

Van der Graaff N A, P Baas (1974) Wood anatomical variation in relation to latitude and altitude. Blumea 22:101-121.

Zimmermann M H (1983) Xylem Structure and the Ascent of Sap. Springer-Verlag, Berlin. Alemania. 143 p. 